Ann. Zootech., I977, 26 (I), 59-68.

\title{
Utilisation d'un anticoccidien (Monensin) dans la ration des ruminants 1. - Effets sur les performances d'engraissement des taurillons
}

\author{
Y. GEAY et C. BÉRANGER \\ Laboratoire de la Production de Viande, \\ Centre de Recherches de Clermont-Ferrand, I.N.R.A. \\ St-Genès-Champanelle 63110 Beaumont (France)
}

\begin{abstract}
Résumé
Nous avons comparé la croissance, la composition des carcasses et l'efficacité alimentaire de 36 jeunes bovins mâles à l'engrais recevant une ration condensée (broyée et agglomérée) comportant 0,20 ou 4 o p.p.m. de "Monensin " (un anticoccidien produit à partir du Streptomyces cinnamonensis).

La ration comportait une faible part de céréales ( 13 p. roo) associée à du tourteau de soja en complément de luzerne (25 p. 100) et de pulpes de betteraves (50 p. I00) déshydratées.

L'adjonction de "Monensin " à la ration a entraîné une diminution immédiate, constante et significative $(P<0,05)$ des quantités ingérées. Toutefois, les gains de poids vif et la composition des carcasses ont été semblables d'un lot à l'autre.

Par suite, l'efficacité alimentaire de la ration a été fortement améliorée, d'autant plus que le taux de "Monensin " était plus élevé : + 7,5 p. Ioo pour 20 p.p.m. de "Monensin ", + 5 p. roo pour $4^{\circ}$ p.p.m.
\end{abstract}

\section{Introduction}

Le "Monensin ", produit à partir du Streptomyces cinnamonensis (Haney et Новнм, I967), a été tout d'abord utilisé en aviculture pour ses propriétés anticoccidiennes. Sa propriété d'accroître la proportion d'acide propionique dans le rumen in vitro et in vivo ayant été mise en évidence (RICHARDSON et al., I974), il a été étudié aux États-Unis où on a montré qu'il diminuait le coût alimentaire du gain de poids vif des bouvillons à l'engrais, recevant des rations riches en céréales (CAMPBell et al., I973 a; RAun et al., I974; Wilson, HATField et Hixon, i975; Perry, Beeson et Mohler, I975; Davis et Erhart, I975; Harvey, LinneRUD et TUGMAN, I976) ou améliorait le gain de poids journalier des bouvillons au pâturage (CAmpbell et al., 1973 b; Potter et al., I974; Oliver, I975). 
L'accroissement de la production d'acide propionique qui permet une amélioration de l'efficacité alimentaire, étant particulièrement souhaitable lorsque les animaux reçoivent des rations pauvres en céréales, nous avons cherché, au cours de cette expérience, à préciser les effets de deux taux de "Monensin " dans une ration riche en pulpes de betteraves et ne comportant que I5 p. Ioo de céréales. sur les performances d'engraissement de taurillons.

\section{Matériel et méthodes}

36 taurillons ( 15 Salers et 2 I Charolais $\times$ Salers), âgés de Io mois, et pesant $287 \pm 23 \mathrm{~kg}$ en moyenne, ont reçu à volonté, une ration condensée (broyée et agglomérée) comportant $50 \mathrm{p}$. Ioo de pulpes de betteraves déshydratées, $25 \mathrm{p}$. Ioo de luzerne déshydratée et $25 \mathrm{p}$. roo de concentré (54 p. Ioo de maïs grain, 40 p. Ioo de tourteau de soja et $6 \mathrm{p}$. Ioo de complément minéral). Les 3 aliments (pulpes, luzerne et concentré) fabriqués séparément, ont été mélangés chaque jour avant leur distribution.

Durant 3 semaines, l'ensemble des animaux, qui recevait au préalable du foin et $2 \mathrm{~kg}$ de concentré, s'est adapté à cette ration témoin. A la fin de cette période préexpérimentale, les taurillons ont été répartis, au sein de chaque type génétique, en blocs de 3 animaux, sur la base du poids vif, de l'âge et de la croissance durant ces 3 semaines. A l'intérieur de chacun des blocs, les 3 animaux ont alors reçu respectivement par $\mathrm{kg}$ de ration : o (lot I), 20 (lot II) et 40 p.p.m. (lot III) de "Monensin ". Celui-ci a été incorporé à l'aliment concentré, à raison de $80 \mathrm{mg} / \mathrm{kg}$ pour le lot II (20 p.p.m.) et de I6o mg/kg pour le lot III (40 p.p.m.).

La période préexpérimentale de 3 semaines, au cours de laquelle les animaux ont reçu la ration témoin, a eu pour objectif de séparer l'effet du changement de régime (foin puis aliments condensés) s'il devait exister, de l'adjonction de "Monensin "sur l'appétit des animaux.

Les animaux, placés en stabulation entravée, ont été alimentés individuellement. Leurs consommations journalières ont été mesurées (5 jours par semaine). Ils ont été pesés deux jours consécutifs à la même heure : avant la période préexpérimentale d'adaptation à la ration condensée (sur le régime de foin), à la fin de cette période préexpérimentale, à la fin d'une période de 3 semaines d'adaptation au "Monensin ", puis toutes les 4 semaines au cours de la période expérimentale et enfin la veille du départ pour l'abattoir.

Pour calculer le gain de poids vif, total et journalier des animaux au cours des deux périodes d'alimentation (préexpérimentale et expérimentale), nous avons utilisé les données corrigées obtenues à partir de l'équation de régression des poids $(\mathrm{P})$ en fonction du temps $(\mathrm{t})$ selon une fonction de la forme :

$$
\mathrm{P}=a+b t+c t^{2}+d t^{3}
$$

Les animaux ont été abattus par blocs de 3 , en moyenne au bout de 182 jours de période expérimentale. A l'abattage, les poids des différents éléments du corps entier ont été mesurés et la composition tissulaire de la carcasse a été estimée à partir de celle du morceau monocostal prélevé au niveau de la i I $^{\mathrm{e}}$ côte (RoBelin et GEAY, I975). 


\section{Résultats}

Au cours de la période expérimentale, un animal du lot II (20 p.p.m. de "Monensin ") a été accidenté et a dû être abattu. Les résultats de ce lot ont donc été obtenus à partir de II animaux seulement.

\section{Croissance}

Aucun trouble n'a perturbé la croissance des animaux. Celle-ci a été non significativement différente entre lots durant la période préexpérimentale. Durant les 183 jours de la période expérimentale, le gain de poids vif des animaux recevant les divers taux de "Monensin " a été satisfaisant : I 243 et $\mathrm{I} 235 \mathrm{~g} / \mathrm{j}$ respectivement pour le lot II (20 p.p.m.) et le lot III (40 p.p.m.), et pratiquement identique à celui des animaux recevant la ration témoin (I $235 \mathrm{~g} / \mathrm{j}$ ) (tabl. I) (fig. I).

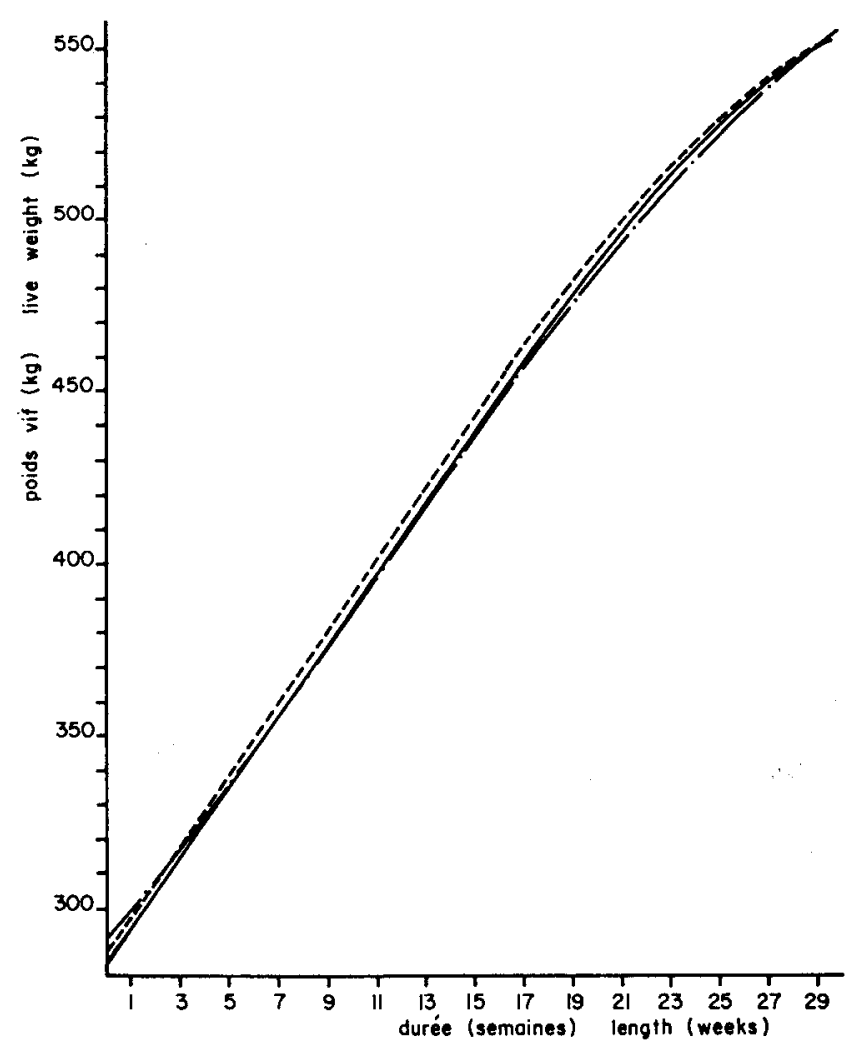

FıG. I. - Évolution du poids vif au cours de la période d'engraissement Evolution of the live weight during the fattening period.

Lot I témoin (control)

Lot II (20 p.p.m.)

- L Lot III (40 p.p.m.) 
TABLEAU I

Poids, gains de poids et quantités ingérées par les taurillons au cours des périodes préexpérimentale et expérimentale

Weight, weight gain and feed intake of young bulls during pre-experimental and experimental periods

\begin{tabular}{|c|c|c|c|}
\hline Lots $\mathrm{n}^{\circ}\left(\right.$ Group $\left.n^{\circ}\right)$ & I & II & III \\
\hline $\begin{array}{l}\text { Teneur en Monensin de la ration (p.p.m.) } \\
\text { (Monensin content of the diet) }\end{array}$ & $\mathbf{o}$ & 20 & $4^{\circ}$ \\
\hline Nombre d'animaux (Number of animals) & 12 & I I & 12 \\
\hline $\begin{array}{l}\text { Période préexpérimentale }\left(\begin{array}{ll}2 I & j\end{array}\right) \text { (Pre-expe- } \\
\quad \text { rimental period }(2 x d))\end{array}$ & & & \\
\hline Poids initial $(\mathrm{kg}$ ) (Initial weight).$\ldots$ & $287 \pm 22$ & $282 \pm 25$ & $288 \pm 21$ \\
\hline Poids final $(\mathrm{kg})$ (Final weight)..... & $315 \pm 19$ & 3 II \pm 23 & $314 \pm 20$ \\
\hline Gain journalier (g) (Daily gain) $\cdot \cdots$ & I 333 & I $38 \mathrm{I}$ & I 238 \\
\hline \multicolumn{4}{|l|}{$\begin{array}{l}\text { Matière sèche ingérée } \mathrm{kg} / \mathrm{j} \text { (p. Ioo) (Dry) } \\
\text { matter intake) : }\end{array}$} \\
\hline & & & \\
\hline lucerne) & $I, 4(25,2)$ & $1,4(25,6)$ & I, $5(25,8)$ \\
\hline 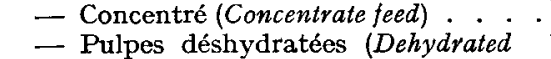 & $1,4(25,2)$ & $I, 3(24,5)$ & $1,4(24,5)$ \\
\hline $\begin{array}{l}\text { - Pulpes déshydratées (Denydrated } \\
\text { pulps).......... }\end{array}$ & $2,8(49,5)$ & $2,7(49,9)$ & $2,8(49,7)$ \\
\hline _- Totale (Total) . . . . & 5,6 & 5,4 & 5.7 \\
\hline - Par kg de gain (Per kg gain) & 4,2 & 3.9 & 4,6 \\
\hline $\begin{array}{l}\text { - Par kg poids métabolique (g) (Per kg } \\
\text { metabolic weight) } . . . .\end{array}$ & 77 & & \\
\hline \multicolumn{4}{|l|}{ Période expérimentale : } \\
\hline Durée (j) (Time $(d)) \quad \ldots$ & $\mathrm{I} 83 \pm 2 \mathrm{I}$ & $181 \pm 22$ & $\mathrm{I} 83 \pm 2 \mathrm{r}$ \\
\hline Poids initial (kg) (Initial weight) & $315 \pm 19$ & $3 \mathrm{II} \pm 23$ & $314 \pm 20$ \\
\hline Poids final (kg) (Final weight). & $54 \mathrm{I} \pm 2 \mathrm{I}$ & $536 \pm 33$ & $54^{\circ} \pm 27$ \\
\hline Gain journalier (g) (Daily gain) $\cdot \cdot \cdot \cdot$. & I 235 & I 243 & I 235 \\
\hline \multicolumn{4}{|l|}{$\begin{array}{l}\text { Matière sèche ingérée } \mathrm{kg} / \mathrm{j} \text { (p. IOO) (Dry } \\
\text { matter intake) : }\end{array}$} \\
\hline $\begin{array}{c}\text { - Luzerne déshydratée (Dehydrated } \\
\text { lucerne) }\end{array}$ & $I, 9(25,6)$ & $1,8(25,5)$ & $1,6(25,6)$ \\
\hline $\begin{array}{l}\text { - Concentré (Feed concentrate) } \\
\text { - Pulpes déshydratées (Dehydrated }\end{array}$ & $1,8(24,6)$ & $1,7(24,7)$ & $I, 6(24,6)$ \\
\hline $\begin{array}{l}\text { Pulpes desnyaratees (Denyaratea } \\
\text { pulps) }\end{array}$ & $3.7(49,8)$ & $3,4(49,8)$ & $3,2(49,8)$ \\
\hline - Totale (Total) $\ldots \ldots$ & 7,4 & 6,9 & 6,4 \\
\hline - Par kg de gain (Per kg gain) & 6,0 & 5,6 & 5,2 \\
\hline $\begin{array}{c}\text { - Par kg poids métabolique }(\text { Per } k g \\
\text { metabolic weight }) . . .\end{array}$ & 79 & 74 & 68 \\
\hline
\end{tabular}

Seules, des différences significatives de gain de poids vif $(\mathrm{P}<0,05)$ sont apparues entre les races ( 8 p. IOo) en faveur des Charolais $\times$ Salers - ce qui rejoint les résultats que nous avons obtenus par ailleurs (GEAY et BERANGER, Ig6g; GEAY, RoBelin, BERANGER, I976) - mais, nous n'avons pas constaté cependant d'interaction significative entre les races et les traitements. 


\section{Quantités d'aliments ingérées}

Au cours des périodes préexpérimentale et expérimentale, les animaux ont bien consommé les 3 aliments : luzerne, pulpes et concentré, dans les proportions prévues dans le protocole. Durant les 3 semaines de la période préexpérimentale, les quantités ingérées par les animaux ont augmenté de façon identique, quel que soit le lot (fig. 2); les animaux s'adaptant à la ration condensée qui ne com-

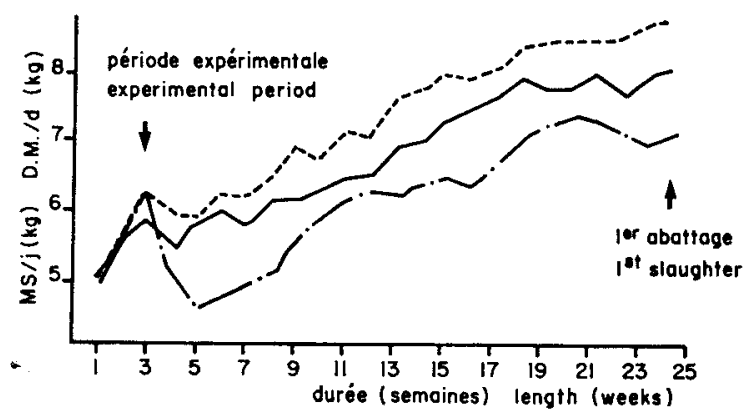

FIG. 2. - Évolution des quantités de matière sèche ingérées par semaine

Evolution of the dry matter intake each day and each week.

portait alors pas de "Monensin ". Les quantités de matière sèche consommée par Ioo $\mathrm{kg}$ de poids vif, par les animaux des lots I et III, étaient alors identiques et peu différentes de celles des animaux du lot II (fig. 3).

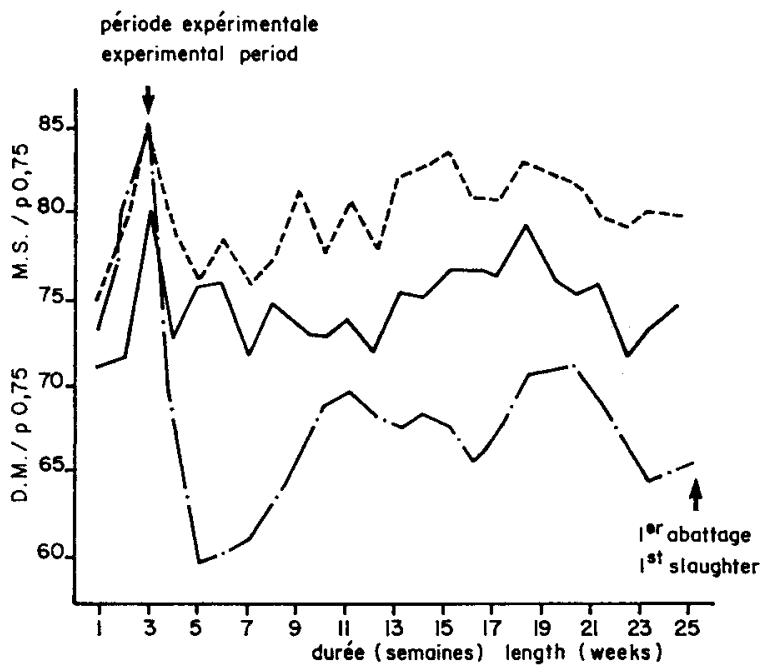

Fig. 3. - Évolution des quantités de matière sèche ingérées par Ioo kg de poids vit Evolution of the dry matter intake per $100 \mathrm{~kg}$ of live weight. 
Dès la première semaine de la période expérimentale, les consommations des rations comportant le "Monensin " ont décru brutalement, plus fortement chez les animaux qui en recevaient 40 p.p.m. que chez ceux qui n'en recevaient que 20 p.p.m. Passé une période (de 27 à I4 jours), elles ont augmenté parallèlement à celles des animaux du lot témoin, jusqu'aux premiers abattages.

Durant toute la période expérimentale, les animaux recevant le "Monensin " ont consommé, à même durée, significativement $(\mathrm{P}<0,05)$ moins de matière sèche que ceux du lot témoin; respectivement 7 et I4 p. Ioo pour le lot II et le lot III (tabl. 3). Par suite, l'efficacité alimentaire de la ration a été améliorée par l'adjonction de "Monensin ": de 7,5 et de I5 p. roo lorsqu'elle en a comporté respectivement 20 et 40 p.p.m (fig. 4 , tabl. 3 ).

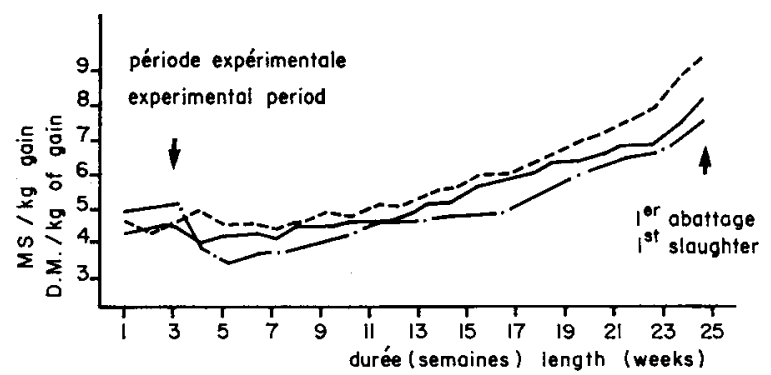

FIG. 4. - Évolution des quantités de matière sèche moyenne ingérées par $k g$ de gain Evolution of the dry matter intake per $k g$ of gain.

Les taurillons Salers ont consommé, à durée constante, une quantité de matière sèche non significativement différente de celle ingérée par les Charolais $\times$ Salers. Nous n'avons pas non plus observé d'interaction significative entre les races et les traitements (tabl. 3).

TABLEAU 2

Poids vif vide, poids et composition de la carcasse des taurillons

Empty live weight, weight and composition of young bull carcasses

\begin{tabular}{|c|c|c|c|}
\hline Lots $n^{0}\left(\right.$ Group $\left.n^{0}\right)$ & I & II & III \\
\hline 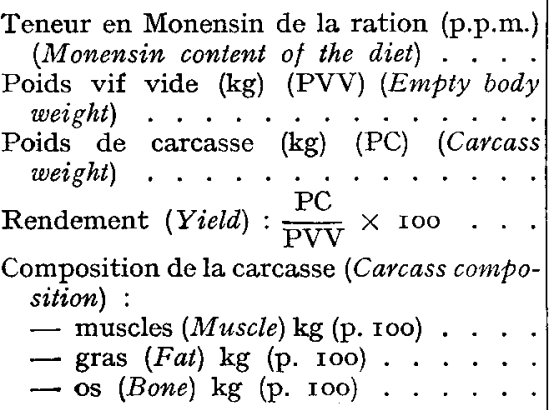 & $\begin{array}{c}o \\
47^{8} \pm \mathrm{I} 8 \\
309 \pm \mathrm{I} 8 \\
64,6\end{array}$ & $\begin{array}{r}229(75) \\
36 \text { (I2) } \\
40(13)\end{array}$ & $\begin{array}{c}40 \\
465 \pm 21 \\
301 \pm 19 \\
64,7\end{array}$ \\
\hline
\end{tabular}


UTILISATION D'UN ANTICOCCIDIEN (MONENSIN)

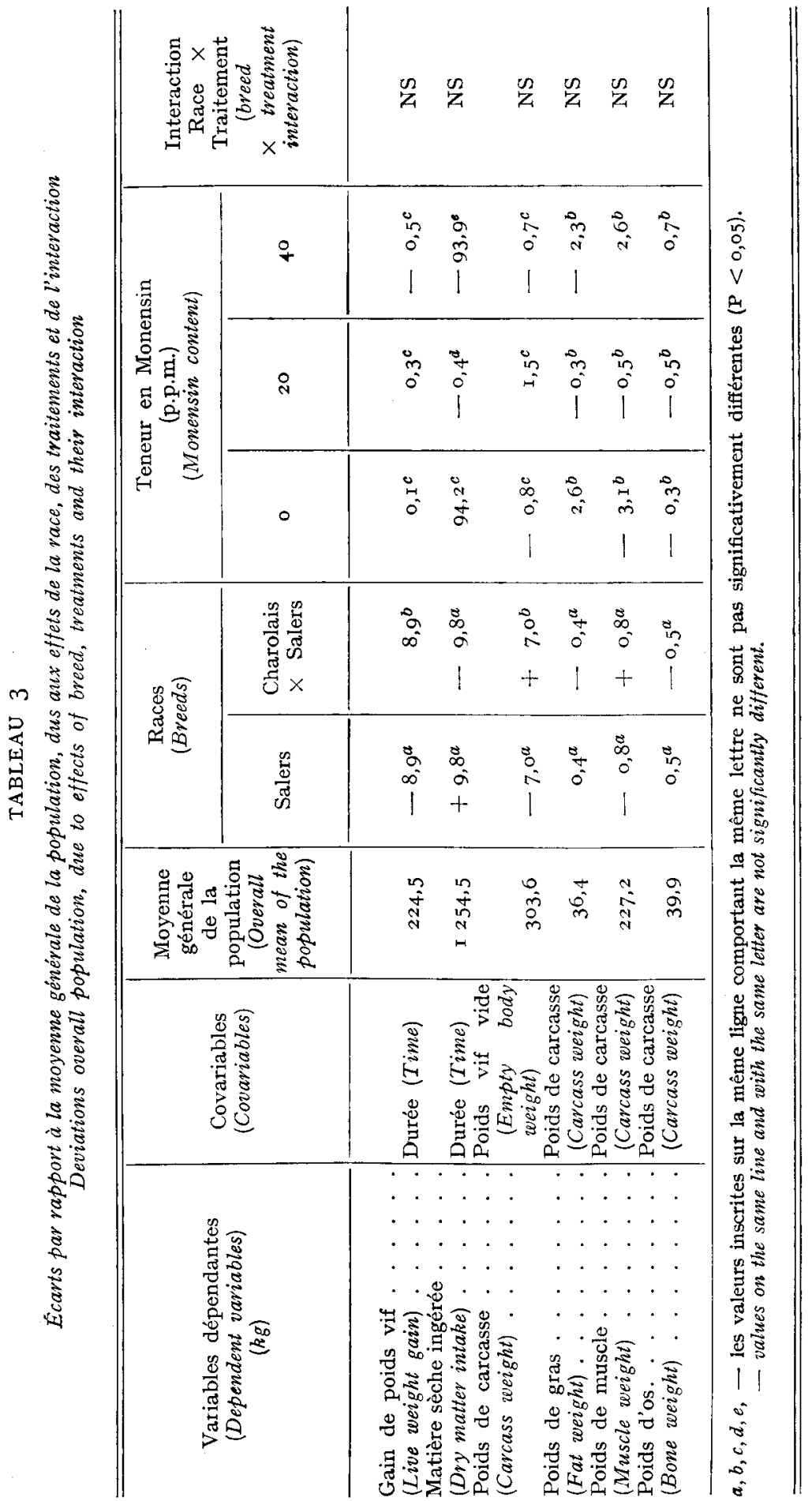




\section{Poids et composition des carcasses}

Les animaux ayant reçu du "Monensin " ont fourni pour un même poids vif, un poids de carcasse non significativement différent de celui des animaux qui n'en recevaient pas et ont eu un même rendement en carcasse : 64,6, 65,0,64,7 respectivement pour les lots I, II et III (tabl. 2). A même poids de carcasse, la composition tissulaire de ces carcasses a été non significativement différente. Cependant, la proportion de muscle a eu tendance à croître et la proportion de gras à décroître avec la quantité de "Monensin " ingéré. La proportion des dépôts adipeux a été très satisfaisante, légèrement plus faible chez les Charolaix $\times$ Salers (Ir,6 p. I00) que chez les Salers, plus précoces (I2,4 p. IOO). Nous n'avons pas non plus observé d'interaction significative entre les races et les traitements à même poids de carcasse (analyse de covariance).

\section{Discussion}

L'incorporation de "Monensin » dans la ration n'a pas provoqué de troubles apparents des animaux, quel qu'ait été son taux d'incorporation. Il n'a pas modifié leur gain de poids vif journalier ainsi que l'ont observé RAUN et al. (I974), EMBRY et SWAN (I975) et HALE et al. (I975), mais contrairement aux résultats de SHERRoD, Kellison et Klett (I975), Bolsen, Corah et Riley (1975) et Utrey, Newton et MCCormick (I975) qui montraient une augmentation significative du gain de poids vif.

Le "Monensin " a entraîné une diminution immédiate, constante et significative des quantités ingérées dans des proportions comparables à celles observées par tous les auteurs précédents. Par suite, l'efficacité alimentaire des animaux a été fortement améliorée, jusqu'à I 5 p. Ioo pour 40 p.p.m. de "Monensin " dans la ration, valeur observée par Davis et ERHART (r975) avec 30 p.p.m. de « Monensin " dans une ration riche en céréales. Enfin, le "Monensin " n'a pas modifié la composition des carcasses. Brown et al. (I974) n'ont également pas observé de modification de la qualité des carcasses, jugées sur leur état d'engraissement apparent, des I I57 bouvillons qu'ils ont utilisés dans leurs expériences.

Ces résultats présentent un intérêt particulier pour la production de taurillons. Lors d'expériences antérieures (GEAY et Beranger, I969; GEAY, I976; GEAY, Robelin, Beranger, I976), nous avons en effet montré qu'une réduction des quantités d'une ration à forte concentration nutritive, distribuée aux taurillons à l'engrais, entraînait une amélioration de l'efficacité alimentaire. Cependant, celle-ci s'accompagnait, à durée constante, selon le génotype, soit d'une diminution importante de l'état d'engraissement à même poids de carcasse, soit d'une diminution du poids de carcasse sans modification de l'état d'engraissement.

L'étude de la digestion de ces rations, entreprise par JoUANY et SENAUd (I977) a mis, en évidence une diminution de la quantité de méthane et une augmentation de la proportion d'acide propionique dans le rumen. Aussi, l'amélioration de l'efficacité alimentaire pourrait-elle s'expliquer, à la fois par un accroissement 
de la métabolisabilité de la ration (diminution des pertes sous forme de méthane), par une élévation du rendement de transformation de l'énergie métabolisable en énergie fixée due à l'augmentation de la proportion d'acide propionique dans le rumen (ARMSTRong et BLAXTER, I957), enfin par une économie des protéines liée à l'utilisation de l'acide propionique comme précurseur du glucose (LENG, STEEL et LUICK, I967). Ce phénomène, peut-être lié au précédent, pourrait également expliquer l'obtention de carcasses légèrement plus maigres.

Reçu pour publication en novembre 1976.

\section{Remerciements}

Cette étude a été réalisée grâce à l'aide de la Société Eli Lilly France, Département Elanco, à qui nous exprimons nos remerciements.

\section{Summary}

Use of an anticoccidial drug (monensin) in the diet of ruminants. I. - Effect on fattening performances of young bulls

In the present study, the effect of adding 0,20 or $40 \mathrm{ppm}$ monensin to a ground and pelleted diet on growth, carcass composition and feed efficiency was studied in 36 fattening young bulls.

The diet contained a small proportion of cereals ( 3 per cent) associated with soyabean meal as a supplement to dehydrated lucerne (25 per cent) and sugar beet pulp (5o per cent).

The young bulls receiving the monensin containing diet had a significantly lower feed intake than the controls. However their daily gain and their carcass composition were not significantly different.

Thus, the feed efficiency of the diet was highly improved with increasing drug levels, i.e. by 7.5 per cent with the diet containing 20 p.p.m. monensin and by 5 p. cent with that containing $4^{\circ}$ p.p.m. monensin.

\section{Références bibliographiques}

Armstrong D. G., Blaxter K. L., I957. The utilization of acetic propionic and butyric acids by fattening sheep. Br. J. Nutr., 11, 4I3-423.

Bolsen K. K., Corah L., Riley J. G., I975. Milo stover silage and monensin for growing calves. J. Anim. Sci., 41, 392 (Abstr.).

Brown H., Carroll L. H., Elliston N. G., Grueter H. P., Mcaskill J. W., Olson R. D. Rathmacher R. P., 1974. Field evaluation of monensin for improving feed efficiency in feedlot cattle. Proc., Western Section, American Society of Animal Science, 25, 300-302.

Campbell L. D., Boling J. A., Bradley N. W. and Lovel D. R., r973a. Antibiotic in finishing rations for steers. Agricultural Experiment Station of the University of Kentucky. 86 th Annual Report. Dec. $3 \mathbf{I}$.

Camprell L. D., Bradeey N. W., Boling J. A., Lovel D. R., I973b. Supplementing antibiotic to grazing steers. University of Ky. Agr. Exp. Sta. 85th Annu. Rep. P. 40.

Davis G. V. Jr., ERHART A. B., 1975. Effects of feeding monensin and implanting diethylstilbestrol on the performance of finishing steers. Kansas Cattle Feeders Day Report. June 6, 3-6.

EMBRY L. B., SwAN W. S., r975. Effects of monensin on feedlot performance of growing and finishing steers. South Dakota. State University A.S. Series p. I9. 
GeAy Y., 1976. Fitting the diet to the potential of the animal. 1. Animals data in : "Improving the nutritional efficiency of beef production ". Proc. Seminar at Clermont-Fernand. October 1975. Commission of the European Communities, Brunds. EUR 5488 e, p. 333 .

Geay Y., Beranger C., I969. Influence de la proportion des céréales dans la ration sur l'état d'engraissement des carcasses de taurillons de I 5 mois. Ann. Zootech., 18, 79-8r.

Geay Y., Robelin J., Beranger C., 1976. Influence du niveau alimentaire sur le gain de poids vif et la composition de la carcasse de taurillons de différentes races. Ann. Zootech., 25, 287298.

Hale W. H., Theurer B., Marchello J. A., Taylor B., Kuhn J., I975. The effect of monensin on growing and finishing cattle. Avizona Cattle Feeders Day, May I. Series. p. 36.

Haney M. E. Jr., Hoenn M. M., 1967. Monensin, a new biologicaly active compound. Antimicrobial Agents and chemotherapy. 349-352.

Harvey R. W., Linnerud A. C., Tugman D. F., i976. Monensin and silage rations for growing cattle. J. Anim. Sci., 42: 275. (Abstr.).

Jouany J. P., Senaud J., 1977. Utilisation d'un anticoccidien (Monensin) dans la ration des Ruminants. II. Effets sur la digestion des aliments dans le rumen. Ann. Zootech. (à paraître).

LENG R. A., Steet J. W., Luick J. R., I967. Contribution of propionate to glucose synthesis in sheep. Biochem. J. I03, $785-790$.

Oliver W. M., I975. Effect of Monensin on gains of steers grazed on coastal bermudagrass. J. Anim. Sci., 41, 999-roor.

Perry T. W., Beeson W. H., Mohler M. T., 1975. Effect of rumensin on beef cattle performance. Purdue cattle feeders day. April 4, 29-33.

Potter E. L., Cooley C. O., Raun A. P., Richardon L. F., Rathmacher R. P., I974. Effect of monensin on daily gain of cattle on pasture. Proc., Western Section. American Society of Animal Science, 25, 343-345.

Raun A. P., Cooley C. P., Rathmacher R. P., Richardon L. F., Potter E. L., I974. Effect of different levels of monensin on feed efficiency ruminal and carcass characteristics of cattle. Proc. Western Section. American Society of Animal Science, 25, 346-349.

Rrchardon L. F., Raun A. P., Potter E. L., Cocoey C. O. and Rathmacher R. P., i974. Effect of monensin upon composition of carcass gain of cattle. J. Anim. Sci., 39, 249 (Abstr.).

Robelin J., GeaY Y., I975. Estimation de la composition des carcasses de jeunes bovins à partir de la composition d'un morceau monocostal prélevé au niveau de la i ${ }^{\mathrm{e}}$ côte. Ann. Zootech., 24, 391-402.

Sherrod L. B., Kellison R. L., Klett R. H., 1975. Monensin levels in growing and finishing steer rations. Texas Tech. University Center at Amarillo. Pantex. Texas. Rsch. Rept., 25, 43-45.

Utley J. R., Newton G. L., McCormick W. C., 1975. Rumensin, Ralgro and Synovex. H. for growing cattle. J. Anim. Sci., 41, 423. (Abstr.).

Wilson L. L., Hatfi eld E. E., Hixon D. I., I975. The effectiveness of different levels of monen$\sin$ on the feedlot performance of finishing steers. Agricultural Experiment Station of the University of Illinois. As. 669 a. Beef cattle day. 